\title{
Simultaneous analysis of major ingredients of Gardenia fruit by HPLC-MS/TQMS method
}

\author{
M.Galaqin ${ }^{1 *}$, K.Uwai², M.Yuguchi², T.Iwasa ${ }^{2}$ \\ ${ }^{1}$ College of Chemistry and Environmental Science, Inner Mongolia Normal University, Hohhot 010022, China \\ ${ }^{2}$ Muroran Institute of Technology, Hokkaido Muroran 050-8585, Japan
}

\author{
ARTICLE INFO: Received: 2 Feb, 2016; Revised: 22 Nov, 2016; Accepted: 7 Dec, 2016
}

\begin{abstract}
An efficient, accurate HPLC-MS/TQMS method was introduced for the quantitative/qualitative simultaneous analysis of main ingredients, namely geniposide and genipingentiobioside, in the Gardenia fruit. The separation was successfully obtained using a $\mathrm{C}_{8}\left(100 \mathrm{~mm} \times 2.1 \mathrm{~mm}, 5 \mu \mathrm{m}, 30^{\circ} \mathrm{C}\right)$ column by gradient elution with ultrapure water as mobile phase, where flow rate was set to $0.2 \mathrm{ml} / \mathrm{min}$ and detection wavelength at $240 \mathrm{~nm}$. The analytical method was validated and the quantification of active compounds, namely genipingentiobioside and gardenoside, was performed. Linearity, precision, repeatability, stability and recovery were also reported. The quantitative analysis revealed that both main ingredients as geniposide and genipingentiobioside have performed a good linear relationship in $0.1-100 \mathrm{mg} / \mathrm{ml}$ concentration range $(r=1.00000$ and $r=0.99998)$. The average content was measured to be $4.842 \%$ with RSD $0.96 \%$ for geniposide and $1.1976 \%$ with RSD $0.47 \%$ for genipingentiobioside in the Gardenia fruit. Accordingly, this method would be feasible for the quantity and quality control of crude drugs.
\end{abstract}

Keywords: HPLC-MS/TQMS, geniposide, genipingentiobioside, quantity control

Copyright $\odot 2016$ Galaqin M, et al. This is an open access article distributed under the Creative Commons Attribution 4.0 International License CC BY, which permits unrestricted use, distribution, and reproduction in any medium, provided the original work is properly cited.

\section{INTRODUCTION}

The dried ripe fruit of Gardenia jasminoides Ellis, a Rubiaceae family, has been wildly used in Mongolian, Chinese and Kampo medicine. Now it becomes a hot topic due to its numerous good effects, such as on the treatment of irritability in febrile diseases, jaundice, acute conjunctivitis, epitasis, haematuria, pyogenic infections and ulcers of the skin, sprains and painful swellings due to blood stasis [1-7]. It contains mainly iridoid, such as geniposide, genipingentiobioside, gardenoside and geniposidic acid, etc. [8]. These ingredients were believed to be the pharmaceutically active components of this fruit.

HPLC-MS/TQMS technology has unique advantages in the research of chemical composition, quality standard and fingerprint of traditional Chinese medicine $[9,10]$. Recently, many researchers have focused on the HPLC measurement of the amount of Gardenia acid, genipin glycoside geniposide, crocin and geniposide in the Gardenia [11-14] and qualitative analysis of Gardenia chemical compositions [15-17].

However, the research results on liquid chromatography and mass spectrometry simultaneous determination of geniposide, genipin melibiose and gardenoside in the Gardenia for qualitative and quantitative analysis are not been reported.

The authors, using a high performance liquid chromatography and mass spectrometry (HPLC-MS/ MS), have determined simultaneously the active chemical components in Gardenia fruit. Having analyzed the retention time of the chromatographic peaks, primary/

*corresponding author: e-mail address: gaman@imnu.edu.cn DOI: http://dx.doi.org/10.5564/mjc.v17i43.744 secondarymass spectra and comparison with the reference standards, both quality and quantity of two active components, namely, geniposide and genipingentiobioside have been analyzed. Acomprehensive validation study was also carried out on linearity, precision, repeatability, stability and recovery. The developed HPLC-MS/TQMS method would be helpful for the quality control of crude drugs.

\section{EXPERIMENTAL}

Sample preparation: The dry fruits of Gardenia purchased from Inner Mongolia were ground to powder and passed through a 60-mesh sieve. For extracting the active components, $0.5 \mathrm{~g}$ powders was added in $10 \mathrm{ml}$ ultrapure water and treated with ultrasonic homogenizer $(70 \mathrm{~W}, 20 \mathrm{kHz}$ ) for $30 \mathrm{~min}$. The mixture was centrifuged (3000 rpm) and the supernatant was filtered with $0.45 \mu \mathrm{m}$ porous membrane for analysis. After that, the solution was diluted 1000 times for HPLC measurement.

Preparation of reference solutions: Three reference solutions containing $1 \mathrm{mg} / \mathrm{ml}$ of geniposide (code No. 00007070-212, Wako, Japan) or $1 \mathrm{mg} / \mathrm{ml}$ of genipingentiobioside (code No. 130527, Sichuan Wei Keqi Biotechnology Co., Ltd., China) and their equal mixture (1:1) in ultrapure water were also prepared. The solutions were diluted 100 times again before HPLC measurement.

HPLC analysis: Prepared solutions were analyzed on a Prominence LC-20A (SHIMADZU, Japan), a triple quadrupole mass spectrometry (TQ-MS) combined with a liquid Chromatography Mass Spectrometer system (API2000 LC-MS; AB SCIEX, America). HPLC conditions were as following: the chromatography column is ACE $\mathrm{C}_{8}(100 \mathrm{~mm} \times 2.1 \mathrm{~mm}, 5 \mu \mathrm{m})$; it was adapted gradient with methanol-water solution for mobile phase; the flow 
rate is $0.2 \mathrm{ml} / \mathrm{min}$; the detection wavelength is $240 \mathrm{~nm}$; the column temperature is $30^{\circ} \mathrm{C}$; the injected volume of sample is $0.5 \mu \mathrm{l}$. Conditions for the MS spectrum was as following: the ionization source is electrospray ionization (ESI); the scan patterns is positive ion mode; the scanned area is $10-1100 \mathrm{~m} / \mathrm{z}$; capillary voltage is $5500 \mathrm{~V}$ for LC-MS and $4500 \mathrm{~V}$ for MS/MS; the nebulizer pressure is $50 \mathrm{psi}$ for LC-MS and 17 psi for MS/MS; the gas temperature is $300^{\circ} \mathrm{C}$; the voltage of pieces: $120 \mathrm{~V}$. Experimental data was processed by Analyst 1.4.2 (AB SCIEX, America).

\section{RESULTS AND DISCUSSIONS}

Qualitative analysis of MS determination: The extract of Gardenia jasminoides fruits was injected into API2000 for the MS measurement. Figure 1 shows that the masscharge ratio of main peaks which are $410.9 \mathrm{~m} / \mathrm{z}$, amu (peak 1), $572.7 \mathrm{~m} / \mathrm{z}$, amu (peak 2) and $426.9 \mathrm{~m} / \mathrm{z}$, amu (peak 3).

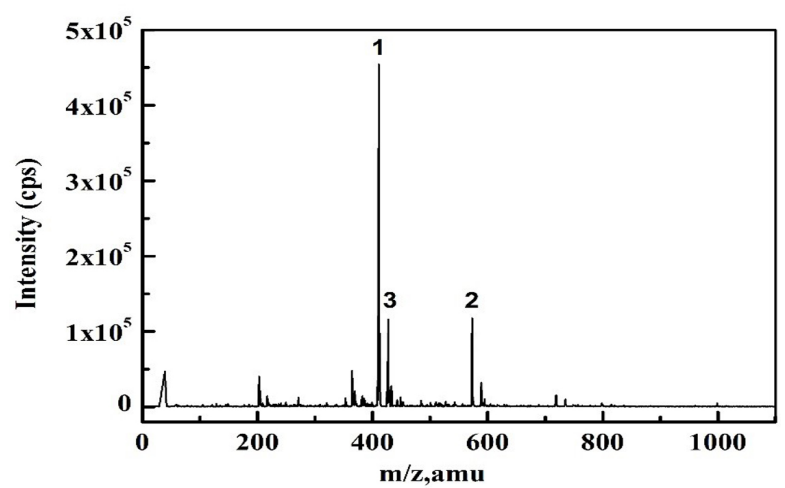

Fig.1. Qualitative analysis of extract of Gardenia fruit

Reference solutions of geniposide and genipingentiobioside were injected into API2000 separately for MS measurement. Geniposide $(\mathrm{Mm}=388.1)$ and genipingentiobioside $(\mathrm{Mm}=550.2)$ are $410.8 \mathrm{~m} / \mathrm{z}$, amu $(\mathrm{M}+\mathrm{X}=388.1+22.7)$ and $572.9 \mathrm{~m} / \mathrm{z}$, amu $(\mathrm{M}+\mathrm{X}=550.2+22.7)$, respectively, as shown in Figure $2 \mathrm{~A}$ and $2 \mathrm{~B}$.

In the MS/MS measurement of quasi-molecular ion peak of reference solution, an ion with mass to charge ratio of $22.8 \pm 0.5 \mathrm{~m} / \mathrm{z}$, amu was detected in the secondary mass spectrometry fragment ions for both references, suggesting $\mathrm{X}$ is estimated to be $\mathrm{Na}^{+}$.

Quantitative analysis by HPLC: The extract of Gardenia jasminoides fruits and reference solution of the mixture of geniposide and genipingentiobioside $(0.5 \mu \mathrm{l}$ each) were injected into the instrument for $\mathrm{LC}$ analysis. Retention times of the detected peaks 1,2 and 3 for the extract were 15.23

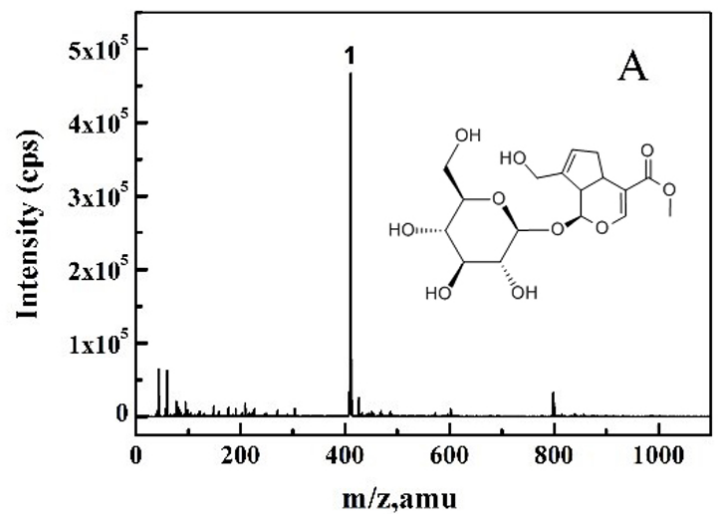

min, 14.16 min and $11.71 \mathrm{~min}$, respectively, as shown in Figure 3B. Among them, the retention times for peak 1 and peak 2 were in consistent with that of peak 1 (geniposide) and peak 2 (genipingentiobioside) of the reference solution (Figure 3A), suggesting the two components detected in Gardenia jasminoides fruits should be geniposide and genipingentiobioside. The extract and references mixture $0.5 \mu$ l were injected six times into the HPLC instrument and the area of chromatographic peaks were determined for calculate the content of them by using external standard method. The average content of geniposide was $4.842 \%$ with relative standard deviation (RSD) $0.96 \%$ and the genipingentiobioside was $1.1976 \%$ with RSD $0.47 \%$, respectively.

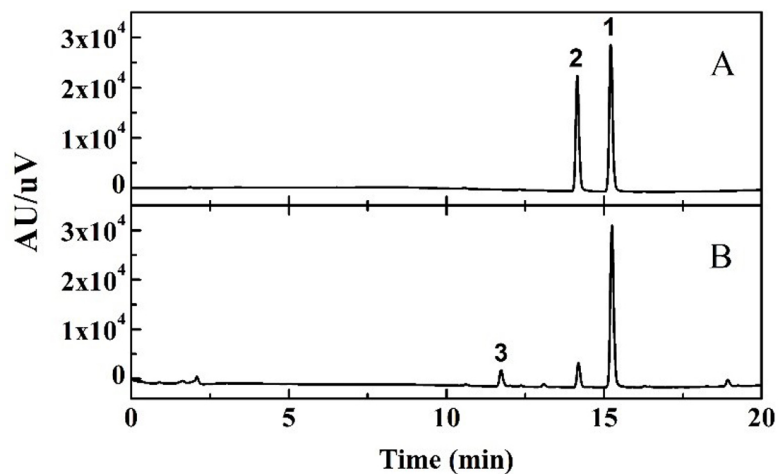

Fig. 3. HPLC chromatograms of extract of Gardenia fruit and reference solution. A: Extract of Gardenia fruit; B: Mixture of geniposide (peak1) and genipingentiobioside (peak2)

\section{Method validation: Linearity}

Linearity range of response was determined for the mixture of geniposide and genipingentiobioside reference solution. Linearity was determined on five level of concentrations as $0.1,1,5,50$ and $100 \mathrm{mg} / \mathrm{ml}$ with three parallel injections for each level by using ultrapure water.

A standard curve was drawn with concentration of reference solution on the $x$ axis, the intensity of chromatographic peak area as vertical coordinate $(y)$.

The linear regression equation of geniposide and genipingentiobioside and its correlation coefficients $(r)$ were obtained as following:

$y=22863 x-1045.2, r=1.00000$ for geniposide;

$y=18471 x-3431.9, r=0.99998$ for genipingentiobioside;

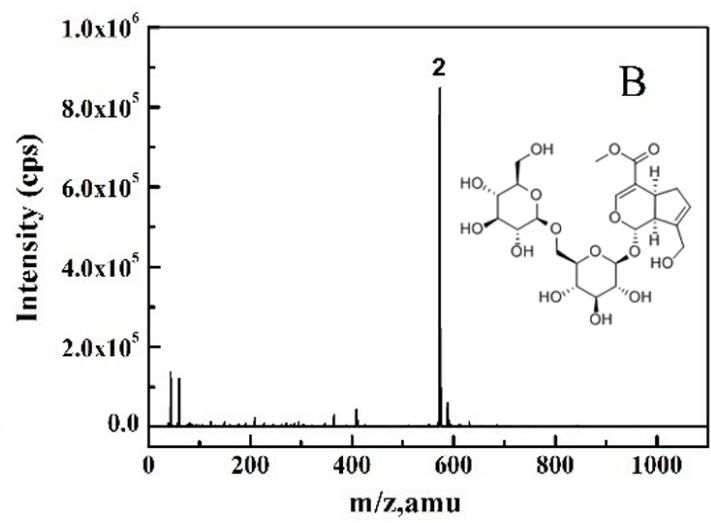

Fig. 2. Mass Spectra of reference solutions, A: TIC of geniposide, peak 1: $410.9 \mathrm{~m} / \mathrm{z}$, amu; $\mathrm{B}$ : TIC of genipingentiobioside, peak 2: $572.7 \mathrm{~m} / \mathrm{z}$, amu 
It can be seen that both geniposide and genipingentiobioside have showed good linear relationship in the range from 0.1 to $100 \mathrm{mg} / \mathrm{ml}$, which indicated that analytical method performance indicators meet the verification requirements of the quantitative analysis method.

Extract Stability: Extract of gardenia fruits $(0.5 \mu \mathrm{l})$ were solubilized in ultrapure water before the analysis and the stability was evaluated at room temperature every $2 \mathrm{~h}$, up to $12 \mathrm{~h}$. The average peak area of geniposide was 68500 with RSD of $0.21 \%$, and genipingentiobioside was 7417 with RS.D of $1.22 \%$, which indicate that the extract were found to be stable in water at room temperature for at least $12 \mathrm{~h}$. Precision of the sample preparation: To evaluate the precision of the sample preparation, five solutions at $0.5 \mu \mathrm{l}$ in ultrapure water were prepared. The estimated RSD of average peak area was $0.18 \%$ for geniposide and $0.70 \%$ for genipingentiobioside, which indicated the well precision of the sample preparation.

Repeatability of the method: In order to evaluate the repeatability of the method, six solutions $(0.5 \mu \mathrm{l})$ prepared from the extract of Gardenia fruits injected and the chromatographic peak areas were determined. Then the amount of each injection was calculated in order to estimate the RSD, Geniposide (average content $(n=6)$ $(4.84 \%$ and R.S.D. $0.96 \%)$ and genipingentiobioside (1.20\%, R.S.D. $0.47 \%$ ), respectively.

Recovery: In order to evaluate the percentage recovery of reference solution into extract, $1 \mathrm{ppm}$ of the mixture of geniposide and genipingentiobioside were added into the prepared extract of Gardenia respectively, and then extracted $0.5 \mu \mathrm{l}$ from it to analyze 5 times. The recovery results showed that average spike recoveries and RSD was $109.13 \%$ and $0.30 \%$ for geniposide, and $94.49 \%$ and $0.31 \%$ for genipingentiobioside.

\section{DISCUSSION}

The content of extract with water is the highest among three samples which were extracted with different solvents of ultrapure water. The samples were scanned in the range of $200-650 \mathrm{~nm}$ wavelength by using a spectrophotometer, and the maximum absorption peak of reference solution of geniposide and genipingentiobioside were observed at $238 \mathrm{~nm}$ and $239 \mathrm{~nm}$, respectively. Therefore, these two compounds were detected at $240 \mathrm{~nm}$ wavelength simultaneously in the follow-up tests. The active ingredients of samples can be measured by both $\mathrm{C}_{18}$ and $\mathrm{C}_{8}$ columns which were used in the liquid chromatography. It was found that the $\mathrm{C}_{8}$ column can more improve the efficiency of tests than $\mathrm{C}_{18}$, therefore, the $\mathrm{C}_{8}$ column was selected. Crude drug extract is an extremely complex mixture with multi chemical compositions. It was difficult to quantify each chemical composition simultaneously by only liquid chromatography, while, liquid chromatography with the mass spectrometer detector can well solve the problem. This method is capable for detecting variety of ingredients in crude drugs with good correspondence.

\section{CONCLUSIONS}

In the present study, we applied HPLC-MS/TQMS method to determine the content of geniposide and genipingentiobioside in Gardenia jasminoides fruits. This determination method is an efficient, accurate and comprehensive evaluation of the active ingredients. Accordingly, this is a feasible method in the crude drug research and quality control.

\section{ACKNOWLEDGEMENTS}

This work is partially supported by International Research Funding of Muroran Institute of Technology.

\section{REFERENCES}

1. Bergonzi M.C., Righeschi C., Lsacchi B., Bilia. A.R. (2012) Identification and quantification of constituents of Gardenia jasminoides Ellis (Zhizi) by HPLC-DADESI-MS. Food Chem, 134, 1199-1204.

2. Cai X.S., Zhang R., Guo Y., He J., Li S., et al. (2015) Optimization of ultrasound-assisted extraction of gardenia fruit oil with bioactive components and their identification and quantification by HPLC-DAD/ESIMS2. Food Funct, 6, 2194-2204.

3. Coran S.A., Mulas S., Vasconi A., (2014) Profiling of components and validated determination of iridoids in Gardenia Jasminoides Ellis fruit by a high-performance-thin-layer-chromatography/mass spectrometry approach. J. Chromatogr A., 1325, 221-226.

4. Han Y., Wen J., Zhou T.T., Fan G. (2015) Chemical fingerprinting of Gardenia jasminoides Ellis by HPLCDAD-ESI-MS combined with chemometrics methods. Food Chem, 188, 648-657.

5. He M.L., Cheng X.W., Chen J.K., Zhou T.S. (2006) Simultaneous determination of five major biologically active ingredients in different parts of Gardenia jasminoides fruits by HPLC with diode-array detection. Chromatographia, 64, 713-717.

6. He W.H., Liu X.A., Xu H.G., et al. (2010) On-line HPLC-ABTS screening and HPLC-DAD-MS/MS identification of free radical scavengers in Gardenia (Gardenia jasminoides Ellis) fruit extracts. Food Chem, 123, 521-528.

7. Zhang X.L., Yu H.J. (2013) Sensitive Determination of Bioactive Crocin Analogues in Gardenia Jasminoides Ellis using SPE prior to HPLC Analysis. Appl Mech Mater., 303-306, 654-658.

8. Sheng Y. (2013) The Research Status of Traditional Chinese Medicine Gardenia. Journal of Anhui Agri. Sci., 41, 7759-7760+7772.

9. Guo-Yuan Z, Guang-Ying C, Qing-Yang L. (2004) HPLC/MS/MS Method for Chemical Profiling of Radix Peucedani(Baihua Quianhu). Chin JNatMed., 2, 51-55.

10. Yu J., Shanshan X., Yuqing S. (2003) High Performance Liquid Chromatography/Photodiode Array Detection/Mass Spectrometry 2 (HPLC/DAD/MS2) Identification of Ingredients of Banlangen Injection. Chinese J.Chromatography, 21, 558-561.

11. Bing H., Ji T., Chun-hong L. (2010) Content variation of 4 mainly components in Fructus Gardeniae of different maturity and different parts. Chin. J. Pharm. Anal., 30, 801-805.

12. Chen S., Hong W., Huan W., Teng Yue. (2012) Content determination of geniposide in Fructus Gardeniae and Baked Fructus Gardeniae Using Ultra performance liquid Chromatography. J. AHHUI TCM College, 31, 56-59.

13. Qi Z., Xiao-xia S., Bing-zhong W. (2012) Influences 
of Different Origins and Collection Time on Content of Geniposide in Gardeniae Fructus. Modern Chinese Medicine, 14, 18-20.

14. Qunlian H., Can T., Yanyan Z. (2011) Content Determination of Geniposide in Fructus Gardeniae by HPLC. China Pharmaceuticals, 20, 20-22.

15. Xiao-Mei F., Gui-Xin C., Zheng-Tao W. (2008) Chemical Constituents of Gardenia jasminoides Ellis. Chin J Nat Med., 6, 418-420.
16. Xiao-yan W., Li Z., Tian-qi W. (2013) Analysis on Chemical Constituents of Gardenia jasminoides by UHPLC-Q-TOFMS. J. Chin. Medic. Mater., 36, 407-410.

17. Yue-ming Z., Zhong-li Z., Ya-qin Y. (2013) Study on the Chemical Components of Gardenia jasminoides. J. Chin. Medic. Mater., 36, 225-227. 